First Trimester Plasma Glucose Values in Women without

Diabetes are Associated with Risk for Congenital Heart

Disease in Offspring

Helle, Emmi I. T.

2018-04

Helle , E I T , Biegley , P, Knowles, J W , Leader , J B , Pendergrass , S , Yang , W , Reaven , G R , Shaw , G M , Ritchie , M \& Priest , J R 2018 , ' First Trimester Plasma Glucose Values in Women without Diabetes are Associated with Risk for Congenital Heart

Disease in Offspring ' , The Journal of Pediatrics , vol. 195 , pp. 275-278 . https://doi.org/10.1016/j.jpeds.2017.10.04

http://hdl.handle.net/10138/301211

https://doi.org/10.1016/j.jpeds.2017.10.046

publishedVersion

Downloaded from Helda, University of Helsinki institutional repository.

This is an electronic reprint of the original article.

This reprint may differ from the original in pagination and typographic detail.

Please cite the original version. 


\title{
First Trimester Plasma Glucose Values in Women without Diabetes are Associated with Risk for Congenital Heart Disease in Offspring
}

\author{
Emmi I. T. Helle, MD, PhD, MSc (Econ) $)^{1,2}$, Preston Biegley, BS ${ }^{3}$, Joshua W. Knowles, MD, PhD ${ }^{1}$, Joseph B. Leader, BA ${ }^{3}$, \\ Sarah Pendergrass, PhD MS ${ }^{3}$, Wei Yang, $\mathrm{MS}^{4}$, Gerald R. Reaven, $\mathrm{MD}^{1}$, Gary M. Shaw, $\mathrm{DrPh}^{4}$, \\ Marylyn Ritchie, $\mathrm{PhD}^{3}$, and James R. Priest, $\mathrm{MD}^{1,4,5}$
}

In a retrospective study of 19171 mother-child dyads, elevated random plasma glucose values during early pregnancy were directly correlated with increased risk for congenital heart disease in offspring. Plasma glucose levels proximal to the period of cardiac development may represent a modifiable risk factor for congenital heart disease in expectant mothers without diabetes. (J Pediatr 2018;195:275-8).

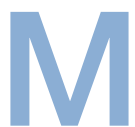

aternal diabetes has long been recognized to be a risk factor for congenital heart disease in offspring. ${ }^{1,2}$

Despite the different pathophysiological mechanisms, both type 1 and type 2 diabetes contribute to the risk of congenital heart disease ${ }^{3}$ and although attenuated in women with improved glucose control, the risk of congeniSee editorial, $\mathbf{p} 9$ and related article, $p 121$

\section{Methods}

We assembled a retrospective multi-institutional cohort of 19107 mother-child dyads of mothers aged 16 years or older for which prenatal information and postnatal diagnoses were available (Stanford Healthcare years 2009-2014 $[\mathrm{n}=9742]$ and Geisinger Health System years tal heart disease does not revert to baseline.

Population studies suggest the presence of acute diabetic complications during pregnancy significantly increase risk of all types of congenital heart diseases in offspring. ${ }^{1}$ Interestingly, a recent case-control study in mothers without diabetes displayed elevated random plasma glucose values during the second trimester in mothers of offspring with tetralogy of Fallot compared with mothers whose offspring did not have congenital heart disease. ${ }^{6}$ These findings suggest that risk of congenital heart disease in the fetus conferred by maternal diabetes may be related to greater variations in plasma glucose levels in mothers with diabetes rather than, to long-term glucose values (glycated hemoglobin A1c), plasma insulin level, or a clinical diagnosis of diabetes. In addition, it raises the question of whether transient plasma glucose elevations even in mothers without diabetes could predispose offspring for congenital heart disease.

Though studies of glucose metabolism performed during the second trimester appear to be strongly linked to glucose metabolism during both the periconceptional period and first trimester, ${ }^{7-9}$ cardiac morphogenesis occurs during the first trimester between gestational weeks $4-10 .{ }^{10}$ Most clinical measurements of glucose metabolism such as the oral glucose tolerance test (OGTT) are typically performed during the second trimester after the heart has already formed. Therefore, we sought to determine if random nonfasting plasma glucose levels measured proximally to the period of cardiac development encompassing the first trimester were related to the risk of congenital heart disease both in mothers with diabetes and without diabetes.

\footnotetext{
BMI Body mass index

ICD-9 International Classification of Diseases, Ninth Revision

OGTT Oral glucose tolerance test
} 2010-2015 [ $\mathrm{n}=9365])$. We excluded offspring with a diagnosis of aneuploidy or 22q11.2 deletion syndrome, multiple pregnancies, and women with extreme body mass index (BMI) measures $(<15$ or $>50)$. Estimated dates of conception were interpolated in a hierarchical fashion from 280 days prior to birth, unless more specific data was available from International Classification of Diseases, Ninth Revision (ICD-9) codes (765.11-765.19) for prematurity status. Given some ambiguity in estimation of exact timing of laboratory measurements during pregnancy, we obtained all random plasma glucose measurements performed between 4 weeks prior to the estimated date of conception until the end of the 14th gestational week, and also results of the 1-hour OGTT typically completed during late second trimester. If multiple measurements were available, we selected the single highest value for analysis as delayed separation of the plasma can decrease the glucose value of the sample. We included plasma glucose measurements in our analysis, and specifically excluded glucose measurements designated as "fasting" or from glucose tolerance tests within the electronic medical record. Because insulin values are rarely measured as a component of routine clinical care, we did not incorporate insulin or other measures of insulin

From the ${ }^{1}$ Cardiovascular Medicine, Stanford University School of Medicine, Stanford, CA; ${ }^{2}$ Children's Hospital, University of Helsinki, Helsinki, Finland; ${ }^{3}$ Department of Biomedical and Translational Informatics, Geisinger Health System, Danville, PA; ${ }^{4}$ Department of Pediatrics; and ${ }^{5}$ Division of Pediatric Cardiology, Stanford University School of Medicine, Stanford, CA

Funded by the National Institutes of Health (NIH) (K99HL130523 to J.P.), Seed Grant from Stanford Cardiovascular Institute (J.P., G.S., J.K.), and the Centers for Disease Control and Prevention, Center of Excellence Award (U01DD001033). E.H. received postdoctoral funding from The Finnish Medical Foundation, Finnish Foundation for Cardiovascular Research, Biomedicum Foundation, Finnish Foundation for Pediatric Research, Orion Research Foundation, Thrasher Research Fund. The authors declare no conflicts of interest.

0022-3476/\$ - see front matter. ( 2017 Elsevier Inc. All rights reserved.

https://doi.org10.1016/j.jpeds.2017.10.046 


\section{Table I. Measured variables by enrollment site}

\begin{tabular}{lrr} 
& & Stanford \\
& $\mathbf{n = 9 7 4 2}$ & $\begin{array}{c}\text { Geisinger } \\
\mathbf{n}=\mathbf{9 3 6 5}\end{array}$ \\
\hline Age, y, median & 31 & 25 \\
Congenital heart disease, $\mathrm{n}(\%$ of total) & $447(4.6 \%)$ & $364(3.9 \%)$ \\
Prepregnancy diabetes, $\mathrm{n}(\%$ of total) & $60(0.6 \%)$ & $14(0.1 \%)$ \\
Prepregnancy BMl, median (n, \% total with measurements) & $28.1(9643,99.0 \%)$ & $25.1(6112,65.2 \%)$ \\
Early plasma glucose mg/dL, median (n, \% total with measurements) & $92(903,9.2 \%)$ & $89(1389,14.8 \%)$ \\
OGTT 1 h value mg/dL, median (n, \% total with measurements) & $122(1825,18.7 \%)$ & $112(7686,82.1 \%)$ \\
More than 1 glucose measurement (n, \% of those with glucose measurements) & $5(0.6 \%)$ & $386(27.8 \%)$ \\
More than 1 OGTT measurement (n, \% of those with glucose measurements) & $306(16.7 \%)$ & $100(1.3 \%)$ \\
\hline
\end{tabular}

resistance within our analysis other than OGTT. A diagnosis of congenital heart disease was defined by the presence of any type of congenital heart disease (ICD-9 codes 745.0747.49) in the offspring. For individuals with OGTT and glucose measures, we performed logistic regression, and adjusted the model for known maternal risk factors for offspring with congenital heart disease, including presence of pregestational diabetes (defined by the presence of ICD- 9 codes 250-250.93), prepregnancy BMI, and maternal age at delivery. In a sensitivity analysis, we excluded mothers with pregestational diabetes. Power calculations were performed assuming an average glucose value of $89.3 \mathrm{mg} / \mathrm{dL}$ with a SD of $6.2 \mathrm{mg} / \mathrm{dL}$, demonstrating that in a group of 200 cases, 2000 controls displayed $80 \%$ power to detect an effect of 0.18 SDs between the 2 groups. Regression models were constructed using the $\mathrm{R}$ language for statistical computing.

\section{Results}

Review of electronic medical records at both study institutions identified 811 congenital heart disease-affected pregnancies and 18296 without congenital heart disease. At the
Stanford site where detailed diagnostic information was available for review, all categories of congenital heart disease were represented with one-half of cases $(49.9 \%, n=223)$ constituting isolated ventricular or atrial septal defects. Relative to the Geisinger cohort, which is a community-based health system, the mothers at the Stanford cohort were older, with higher BMI, and a greater burden of prepregnancy diabetes (Table I). Plasma glucose values were measured at least once in $11.9 \%$, and OGTT in $49.7 \%$ of mothers. Mothers with measured plasma glucose values were younger than mothers without measurements (median 26 vs 28 years), and the same applied for OGTT values (median 26 vs 30 years).

Among the women with relevant clinical measurements, higher plasma glucose (OR 1.08 per $10 \mathrm{mg} / \mathrm{dL}$ increase in glucose, 95\% CI 1.03-1.12, $P<.001$ ) and higher 1-hour OGTT values (OR 1.06, 95\% CI 1.02-1.10, $P=.002$ ) were both associated with increased risk for congenital heart disease offspring in unadjusted logistic models. After adjustment for clinical correlates of glucose metabolism including maternal age, prepregnancy BMI, and the presence of pre-existing maternal diabetes (Table II), higher random first trimester plasma glucose values remained associated with increased risk for congenital heart disease (aOR 1.08 per $10 \mathrm{mg} / \mathrm{dL}$ increase in

Table II. Logistic regression models for glucose and 1 hour OGTT and risk for congenital heart disease in the offspring including and excluding mothers with diabetes

\begin{tabular}{|c|c|c|c|c|}
\hline \multicolumn{5}{|l|}{ Models for glucose } \\
\hline & \multicolumn{2}{|c|}{$\begin{array}{l}\text { Including all mother-infant } \\
\text { dyads }(\mathrm{n}=\mathbf{2 2 3 3})\end{array}$} & \multicolumn{2}{|c|}{$\begin{array}{l}\text { Excluding mother-infant dyads with } \\
\text { pre-existing maternal DM }(n=2204)\end{array}$} \\
\hline & OR (95\% Cl) & $P$ value & OR (95\% Cl) & $P$ value \\
\hline $\begin{array}{l}\text { Glucose }(\mathrm{mg} / \mathrm{dL} / 10) \\
\text { BMI } \\
\text { Maternal age } \\
\text { Maternal DM }\end{array}$ & $\begin{array}{l}1.079(1.023-1.131) \\
1.007(0.976-1.037) \\
1.005(0.972-1.038) \\
0.687(0.085-3.124)\end{array}$ & $\begin{array}{l}.003 \\
.656 \\
.77 \\
.674\end{array}$ & $\begin{array}{c}1.086(1.023-1.143) \\
1.004(0.974-1.035) \\
1.007(0.974-1.040) \\
n / a\end{array}$ & $\begin{array}{l}.003 \\
.775 \\
.685 \\
\mathrm{n} / \mathrm{a}\end{array}$ \\
\hline \multicolumn{5}{|l|}{ Models for OGTT } \\
\hline & \multicolumn{2}{|c|}{$\begin{array}{l}\text { Including all mother-infant } \\
\text { dyads ( } \mathrm{n}=7147)\end{array}$} & \multicolumn{2}{|c|}{$\begin{array}{l}\text { Excluding mother-infant dyads with } \\
\text { pre-existing maternal DM }(n=7142)\end{array}$} \\
\hline & OR (95\% Cl) & $P$ value & OR (95\% Cl) & $P$ value \\
\hline $\begin{array}{l}\text { OGTT } 1 \mathrm{~h}(\mathrm{mg} / \mathrm{dL}) \\
\text { BMl } \\
\text { Maternal age } \\
\text { Maternal DM }\end{array}$ & $\begin{array}{c}1.003(0.998-1.007) \\
1.006(0.986-1.025) \\
1.029(1.009-1.050) \\
0.000(\text { NA-29.120) }\end{array}$ & $\begin{array}{l}.214 \\
.572 \\
.004 \\
.976\end{array}$ & $\begin{array}{c}1.003(0.998-1.007) \\
1.006(0.986-1.025) \\
1.029(1.009-1.050) \\
n / a\end{array}$ & $\begin{array}{l}.214 \\
.572 \\
.004 \\
\mathrm{n} / \mathrm{a}\end{array}$ \\
\hline
\end{tabular}

Model simultaneously included OGTT, maternal prepregnancy BMI, maternal age, and maternal diagnosis for diabetes mellitus (where applicable). 
glucose, 95\% CI 1.02-1.13, $P=.003$ ) and the association with 1-hour OGTT values was elevated but not statistically significant (aOR 1.03, 95\% CI 0.98-1.07, $P=.214$ ). In a sensitivity analysis excluding women with a pre-existing diagnosis of diabetes $(n=74)$, the positive association between a random plasma glucose value with risk for congenital heart disease among offspring was essentially unchanged (aOR 1.09, 95\% CI 1.02-1.14, $P=.003$ ) (Table II).

\section{Discussion}

Our observations demonstrate that higher random plasma glucose values measured during early pregnancy correlate with increased risk for congenital heart disease in offspring of mothers who do not have diabetes. Furthermore, plasma glucose measured during early pregnancy was more associated with risk for congenital heart disease in offspring, compared with the OGTT which is often used to risk-stratify pregnancies for fetal-echocardiographic screening.

Previous clinical observations detect increased risk for congenital heart disease in offspring even in mothers with diabetes with well controlled glucose levels, ${ }^{4,5}$ suggesting that the risk to offspring may be related to the variation in the plasma glucose levels. Although a lower glycated hemoglobin Alc value reflects lower average glucose levels in the long term, fluctuation in glucose levels are likely higher in mothers with diabetes than in mothers without diabetes. Transient high glucose is known to cause persistent epigenetic changes and altered gene expression in vascular cells during subsequent normoglycemia. ${ }^{11}$ As cardiac development occurs early in pregnancy, and many important complex structures are formed during a short period of time, it is possible that even short elevations in glucose levels during critical time points can be enough to disturb cardiac development.

The exact mechanisms by which high glucose may impact cardiac development are not yet clear. Maternal diabetes has been most strongly associated with conotruncal defects, such as tetralogy of Fallot. ${ }^{3}$ High glucose and augmented oxidative stress have been observed to inhibit migration of neural crest derived cells from the neural tube in study animals, leading to congenital heart disease. ${ }^{12,13}$ Thus, if the results from animal studies can be generalized to humans, transient elevations in glucose values during critical periods of cardiac development might potentially disturb the cardiac neural crest migration even in embryos of mothers without diabetes and contribute to the risk of congenital heart disease in the fetus.

Prediabetes is observed in as many as $28 \%$ of women of childbearing age. ${ }^{14}$ Given that increased risk for congenital heart disease continues to be measurable in women without a preexisting diagnosis of diabetes both previously and now more fully in the data reported here, the contribution of maternal prediabetes to risk of congenital heart disease in offspring requires careful assessment in large prospective cohort studies. As plasma glucose levels are correlated with modifiable behaviors such weight, physical activity, and dietary habits, confirming these results in larger studies could provide additional data for pre- and early pregnancy health recommendations as a strategy to optimize the outcomes of pregnancy.

As a retrospective study, these findings are limited by bias inherent in selection of patients with glucose measurements and the ascertainment challenges inherent to working with data obtained from electronic medical records that did not include information on known risk factors for congenital heart disease such as maternal smoking, alcohol consumption, or infection. Early pregnancy plasma glucose measurements were present for only $13 \%$ of the mother-infant dyads, and the clinical reasoning for obtaining the glucose value was unknown, thus, some values may be fasting or from other controlled states. The Geisinger cohort had individuals with multiple glucose measurements, which may be related to a bias toward more readily availability measurements from an integrated inpatientoutpatient electronic medical record. Indeed, the cohorts displayed differences attributable to the different roles that the 2 institutions play within their respective healthcare markets. The older maternal age, higher BMI, higher rates of preexisting diabetes and congenital heart disease are illustrative of a "higher-risk" maternal cohort, which reflects the role of Stanford Healthcare as a regional and statewide quaternary referral center. In contrast, the Geisinger cohort may represent a less selective population with an "average risk" for adverse outcomes. However, glucose values were measured in similar frequencies in pregnancies with or without congenital heart disease (12.3\% of dyads with congenital heart disease, $13.6 \%$ of dyads without congenital heart disease), which does not suggest selection bias related to clinical suspicion of congenital heart disease or any known correlates. The overall size of the cohort was not adequately powered to characterize the risk for specific congenital heart disease malformations. As such, larger prospective studies are necessary to confirm the generalizability of these findings.

A random plasma glucose value performed early in pregnancy may be a marker for risk of congenital heart disease in offspring in mothers without diabetes. Though strongly influenced by common genetic loci, plasma glucose levels are correlated with modifiable behaviors such as weight, physical activity, and dietary habits. If confirmed in large population based studies, plasma glucose values may represent a modifiable risk factor for congenital heart disease in expectant mothers and women planning pregnancy and a biomarker applicable to risk-stratification of pregnant mothers for prenatal echocardiography screening for congenital heart disease in the fetus.

Submitted for publication Jul 11, 2017; last revision received Sep 18, 2017; accepted Oct 18, 2017

Reprint requests: James R. Priest, MD, Division of Pediatric Cardiology, Stanford University School of Medicine, 750 Welch Rd Suite 300, Stanford, CA 94305. E-mail: jpriest@stanford.edu

\section{References}

1. The infants of diabetic mothers. BMJ 1940;1:1064.

2. Rowland TW, Hubbell JP, Nadas AS. Congenital heart disease in infants of diabetic mothers. J Pediatr 1973. 
3. Øyen N, Diaz LJ, Leirgul E, Boyd HA, Priest J, Mathiesen ER, et al Prepregnancy diabetes and offspring risk of congenital heart disease: a nationwide cohort study. Circulation 2016;7:133, 224353.

4. Lisowski LA, Verheijen PM, Copel JA, Kleinman CS, Wassink S, Visser $\mathrm{GH}$, et al. Congenital heart disease in pregnancies complicated by maternal diabetes mellitus. Herz 2010;35:19-26.

5. Evers IM, de Valk HW, Visser GH. Risk of complications of pregnancy in women with type 1 diabetes: nationwide prospective study in the Netherlands. BMJ 2004;328:915.

6. Priest JR, Yang W, Reaven G, Knowles JW, Shaw GM. Maternal midpregnancy glucose levels and risk of congenital heart disease in offspring. JAMA Pediatr 2015;169:1112-6.

7. Riskin-Mashiah S, Damti A, Younes G, Auslander R. Normal fasting plasma glucose levels during pregnancy: a hospital-based study. J Perinat Med 2011;39:209-11.

8. Grewal E, Kansara S, Kachhawa G, Ammini AC, Kriplani A, Aggarwal N, et al. Prediction of gestational diabetes mellitus at 24 to 28 weeks of gestation by using first-trimester insulin sensitivity indices in Asian Indian subjects. Metabolism 2012;61:715-20.
9. Endo S, Maeda K, Suto M, Kaji T, Morine M, Knoshita T, et al. Differences in insulin sensitivity in pregnant women with overweight and gestational diabetes mellitus. Gynecol Endocrinol 2006;22:343-9.

10. Dhanantwari P, Lee E, Krishnan A, Samtani R, Yamada S, Anderson S, et al. Human cardiac development in the first trimester: a high-resolution magnetic resonance imaging and episcopic fluorescence image capture atlas. Circulation 2009;120:343-51.

11. El-Osta A, Brasacchio D, Yao D, Pocai A, Jones PL, Roeder RG, et al. Transient high glucose causes persistent epigenetic changes and altered gene expression during subsequent normoglycemia. J Exp Med 2008;205:240917.

12. Wu Y, Reece EA, Zhong J, Dong D, Shen WB, Harman CR, et al. Type 2 diabetes mellitus induces congenital heart defects in murine embryos by increasing oxidative stress, endoplasmic reticulum stress, and apoptosis. Am J Obstet Gynecol 2016;215:366, e1-366.e10.

13. Suzuki N, Svensson K, Eriksson UJ. High glucose concentration inhibits migration of rat cranial neural crest cells in vitro. Diabetologia 1996;39:40111.

14. Herman W, Rothberg AE. Prevalence of diabetes in the United States. A glimmer of hope? JAMA 2015;314:1005-7. 